\title{
Architecture and Consequent Physiological Properties of the Semitendinosus Muscle in Domestic Goats
}

\author{
CARL GANS, GERALD E. LOEB, AND FRITS DE VREE \\ Department of Biology, The University of Michigan, Ann Arbor, Michigan \\ 48109-1048 (C.G.); Biomedical Engineering Unit, Queen's University, \\ Kingston, Ontario, Canada K7L $3 N 6$ (G.E.L.); Department of Zoology, \\ Universitaire Instelling Antwerpen, Antwerp, Belgium (F.D.V.)
}

\begin{abstract}
Morphological and physiological analyses confirm that the semitendinosus muscle of goats contains two separate compartments in series, each with distinct innervation. These compartments of the muscle are in turn composed of short fibers (approximately four fibers in series in the proximal compartment and seven to eight fibers in the distal compartment) which overlap each other for more than $30 \%$ of their length, with much of the overlapping portions consisting of slender tails that terminate at one-tenth of the midfiber diameter. Groups of fibers are associated into relatively narrow bands that run end-to-end in each compartment. The data suggest that the maximum length of muscle fibers may be limited; even the fibers of parallelfibered muscles may not scale with the dimension of the animal.
\end{abstract}

Physiological and morphological analyses of several elongate and nonpinnate muscles of cats recently confirmed previous suggestions that their fibers did not run from origin to insertion, but rather were compartmentalized (English and Weeks, '87); they have multiple fibers in series within each fascicle (for a review of the literature on previous opinions, see Loeb et al., '87). Even more curious was that staining and chemical teasing of component fibers showed these not to be arranged in a simple end-to-end pattern (as suggested by Gans, '82); rather they overlap significantly. The overlapping ends are attenuated, with their tips tapering to a diameter of a few microns. Previous morphological analysis (Gans and Bock, '65; Gans and de Vree, '87) had suggested that fibers placed end-to-end should generate forces and excursions equivalent to those of continuous fibers. The study of cats indicated that the short fiber pattern in the semitendinosus muscle reflects a physiological limitation on the length of muscle fibers. If fibers became longer, the time for the action potential wave to pass from the site of the motor endplate to the ends of the fiber would exceed the rise time of a contraction twitch. Hence, the center of the fiber would pull on the relaxed ends of the fiber. The much faster conduction times of motoneurons allow this difficulty to be avoided if the separate fibers are placed endto-end and are innervated by branches of the motor axon.

Test of the short fiber hypothesis required the use of larger organisms; if the hypothesis were true, the number of serially arranged fibers would increase with the linear dimension of the muscle. Here we report on adult goats that are much larger than cats, are easily available, and are not endangered. The architecture of their semitendinosus muscle was studied by several morphological techniques. The conduction properties of the muscle were checked physiologically on two preparations, utilizing a newly designed muscle recording array (Chanaud et al.,'87). This permitted a test of the extent to which physiological and morphological data would be congruent.

\section{MATERIALS AND METHODS}

The semitendinosus muscles of four adult goats Capra hircus (contributed by the Antwerp and Detroit Zoological Gardens) were dissected. Fiber length was determined on

\footnotetext{
Address reprint requests to Dr. Carl Gans, Department of Biology, 2127 Kraus Natural Science Building, University of Michigan, Ann Arbor, MI 48109-1048.
} 
TABLE 1. Arrangement of electrode array ${ }^{1}$

\begin{tabular}{|c|c|c|c|}
\hline Strip A & Strip B & Strip C & Strip D \\
\hline a $1-2$ & $1-2$ & $1-2$ & $1-2$ \\
\hline b $2-3$ & $2-3$ & $2-\overline{3}$ & $2-3$ \\
\hline c $3-4$ & $3-4$ & $3-4$ & $3-4$ \\
\hline a $4-5$ & $4-5$ & $4-5$ & $4-5$ \\
\hline b $5-6$ & $5-6$ & $5-6$ & $5-6$ \\
\hline c $6-7$ & $6-7$ & $6-7$ & $6-7$ \\
\hline $\mathrm{a}-$ & - & - & - \\
\hline - & - & - & - \\
\hline b $\quad 37-38$ & $37-38$ & $37-38$ & $37-38$ \\
\hline c $\quad 38-39$ & $38-39$ & $38-39$ & $38-39$ \\
\hline
\end{tabular}

${ }^{1}$ For each record the signals from the numbered contacts are compared as a result of two equivalent stimulations of differential preamplifications between even- and oddnumbered and odd- and even-numbered recording sites, respectively. Because only 13 preamplifiers were available, the stimulation had to be repeated three times (see $a, b, c)$ for each strip. Hence, the generation of the composite diagram of EMG traces for each experiment involved 12 repeated stimulation sessions.

three specimens by chemical dissection with $30 \%$ nitric acid, which was gradually replaced with glycerol (Loeb and Gans, '86). Once the major portions had been separated, fascicles were transferred into elongate glass troughs and portions of the muscle were removed until only a few unbroken fibers remained. Fluid was added and pipetted off the troughs at their ends, and low concentration glycerol solutions were used to facilitate separation of the fiber ends. The process was checked under a dissecting microscope.

The position and number of motor endplates of three entire muscles were checked by the cholinesterase motor end-plate stain of Karnovsky and Roots ('64, as cited in Loeb and Gans, '86). Freshly dissected muscles were exposed to the acetylcholinesterase solution for between 1-2 hr. Once the motor end-plates were noticeable under the dissecting microscope as discrete white spots, the slips of muscle were incubated in $1 \%$ ammonium sulfide, which fixes the reaction and turns the product blackish brown. The samples were then fixed in $10 \%$ formalin for illustration and mechanical dissection. Care had to be taken to avoid too long a reaction time in the acetylcholinesterase stain as the reaction product then tended to diffuse and migrate to the connective tissues adjacent to the fibers. The internal arrangement of motor end-plates was checked by splitting one muscle longitudinally before staining.

The fiber arrangement of two semitendinosus muscles, each from one goat, was tested physiologically. Adult male goats were deeply anesthetized with ketamine-Rompun i.v. (approximately $0.5 \mathrm{ml} / 4 \mathrm{hr}$ ) and phenobarbital (approximately $30-60 \mathrm{mg} / \mathrm{hr}$ ). The left semitendinosus muscle was exposed through the posteromedial surface of the thigh and freed from covering fascia. The deep portion of the muscle was freed so that the nerves entering the muscle became accessible.

An array of electrodes was placed over the posterodistal aspect of the muscle and held in place with modeling clay and moist towels. The array consisted of flexible printed circuit strips, each holding 40 gold electrode contacts spaced at 3-mm intervals (Chanaud et al., '87). Three or four such plastic strips were placed in parallel onto the surface of the exposed muscle, the compliance of the strips allowing good contact with its surface. Conventional ribbon cables connected selected pairs of adjacent contacts to a bank of 13 parallel preamplifiers. Three sets of recordings ( 13 channels at a time) were made from each array so that all 39 adjacent pairs per array were sampled (Table 1). For each set, the sampled sites were spaced over the length of the array; consequently, minor changes in the efficacy of the electrical stimulation or shifts in the position of the array during recording sessions would be readily apparent.

The main nerves to the muscle were cut and their distal ends drawn into bipolar nerve cuff electrodes. This eliminated reflex loops, the only possible source of long latency potentials besides conduction delay in the muscle fibers themselves. The distal nerve ends were stimulated near the threshold levels (cathode closest to muscle, $0.1-\mathrm{msec}$ pulses, about $100 \mu \mathrm{A}$ current) at which some electromyograms (EMGs) could be detected by portions of the array. Attempts to dissect small fascicles from the peripheral nerve for more selective stimulation were unsuccessful due to connective tissue in the nerve. The muscle surface EMGs and a stimulus synchronizaton pulse were recorded on a 5600 Honeywell medium bandpass 14-channel tape recorder (Honeywell $5600,50-$ to $5,000-\mathrm{Hz}$ bandwidth) and later played back to a multitrace oscilloscope and camera. The prints were trimmed and the individual channels pasted into arrays combining them in topographical order (Table 1).

After repeating the procedure in the second goat, the distal end of the semitendinosus muscle was freed and sutured to a wooden strip, permitting the attachment of 


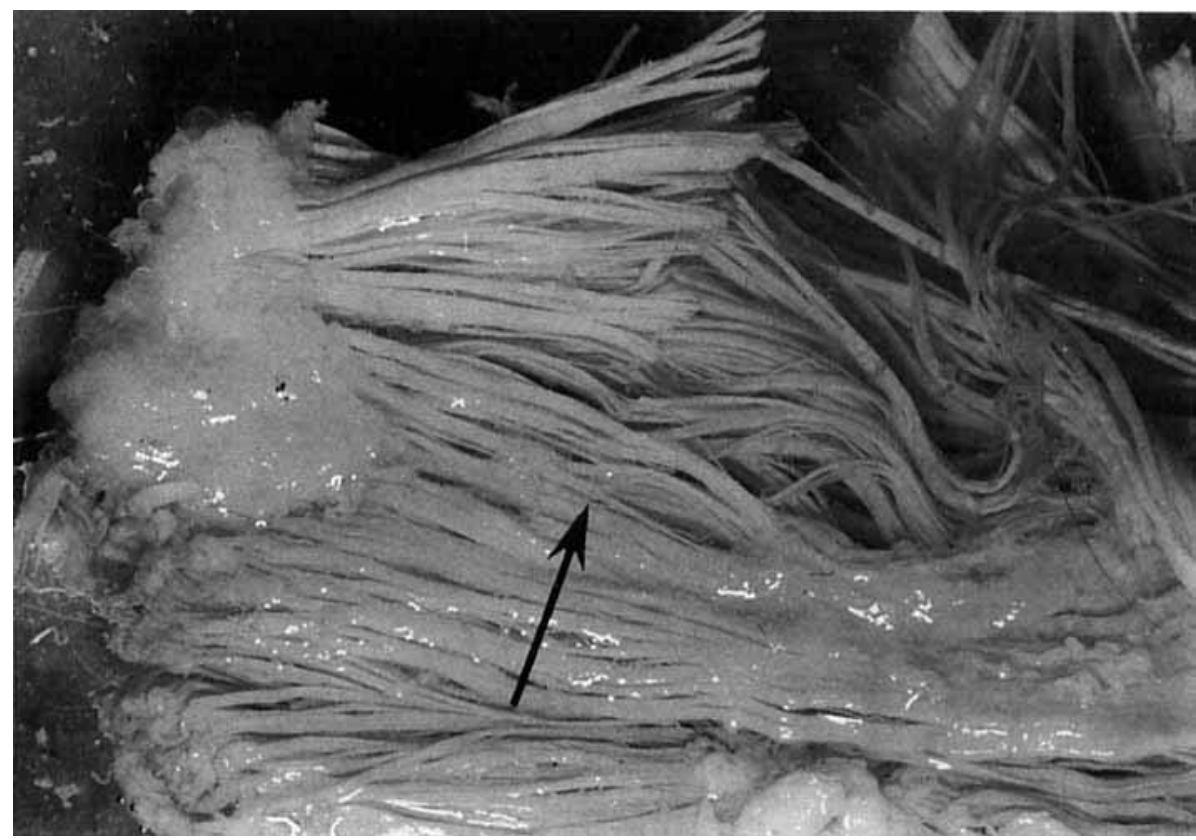

Fig. 1. Capra hircus. Semitendinosus muscle. Detail of the proximal portion of the muscle to illustrate the stage of nitric acid dissection in which the proximal and distal compartments start to break apart (arrow). For part of the muscle, digestion is less complete and the

a 20-kg strain gauge in series with the muscle fibers. This allowed monitoring of the force developed by the muscle.

\section{RESULTS \\ Anatomy}

The semitendinosus muscle is a two-joint muscle that runs from the pelvis to insert on the tibia, extending the hip and flexing the knee. It is a single stout bundle that occupies a diagonal strip along the posterior aspect of the thigh reaching from the free surface of the limb inward toward the femur, so that its sides extend into the muscle mass of the limb along a medial (adjacent to the semimembranosus) and a lateral plane (adjacent to the biceps femoris). The muscle is internally subdivided into a proximal and a distal compartment, the former approximately one-half the length of the latter. A thin, barely noticeable, inclined raphe separates the two (being much less prominent than in the cat). Separate branches of the sciatic nerve reach the thigh in parallel to the main sciatic trunk, each from the medial surface. fascicles still appear continuous. The bundles seen represent major assemblages of multiple fascicles and the blunt terminations of the fibers are discernible under higher magnification. $\times 1.1$.

Exposure to dilute nitric acid first separated the distal and proximal compartments (Fig. 1). Dissolution disclosed two sets of fascicles that were attached end-to-end but then broke cleanly. The fascicles gradually dissolved further, but appeared to consist of single fibers that passed end-to-end within each compartment. Addition of up to 50\% saline to the glycerol solution bathing such digested fascicles caused them to separate further, confirming that each seemingly single fiber actually was composed of multiple, overlapping shorter fibers.

Each section of the muscle contained fibers with one blunt and one finely tapered end and other fibers with two finely tapered ends (Fig. 2). Twelve to 20 randomly selected fibers of each set were measured under a dissecting scope (Table 2). The longitudinal values are approximate, as the fibers showed substantial kinking following the nitric acid digestion (previous unpublished tests indicate that a 10-20\% shrinkage error in longitudinal measurements is common for this technique). The distance over which tapering proceeds is given in Table 3. Measure- 


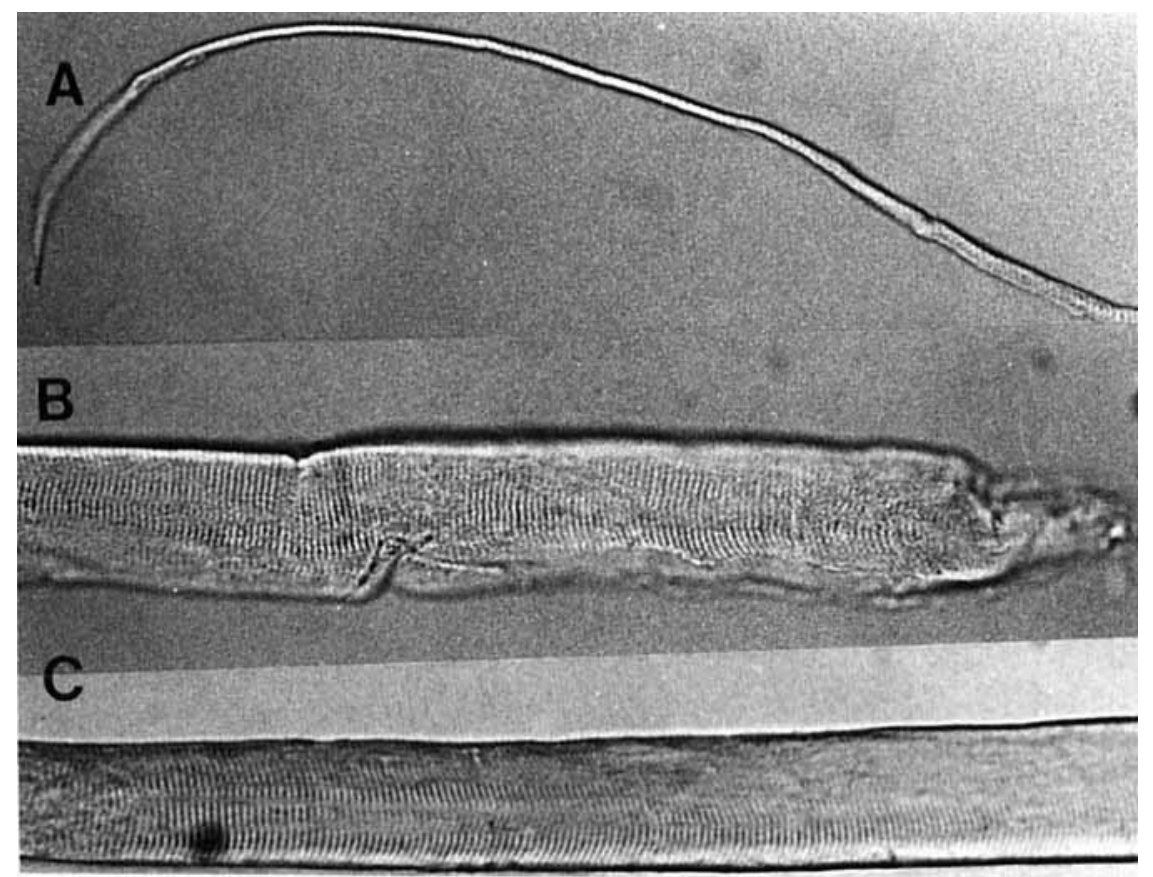

Fig. 2. Capra hircus. Semitendinosus muscle. Three details of a single fiber of the distal compartment taken at a single magnification showing the attenuated end

$T A B L E 2$. Mean values (and ranges) of fiber length $(\mathrm{mm}$ ) from the semitendinosus muscle (total length 158 $\mathrm{mm}$ ) of a goat ${ }^{1,2}$

\begin{tabular}{lcc}
\hline Compartment & 1 blunt end & 2 tapered ends \\
\hline Proximal & $19.92(17.28-23.22)$ & $19.40(16.74-21.96)$ \\
& $\mathrm{N}=20$ & $\mathrm{~N}=17$ \\
Distal & $17.00(15.12-18.25)$ & $17.79(16.04-19.49)$ \\
& $\mathrm{N}=18$ & $\mathrm{~N}=12$ \\
\hline
\end{tabular}

${ }^{1}$ The proximal compartment was approximately $70 \%$ as long as the distal compartment. Fibers with one blunt end attached directly to one of the two terminal tendinous inscriptions. The finely tapered ends interdigitated extensively. Fibers with two fine ends had both interdigitating among adjacent fibers.

${ }^{2}$ In the proximal compartment, mean length did not differ significantly between fibers with one blunt and two fine ends; in the distal compartment, this difference was faintly significant $(P<.05)$. Each mean for the sets of fibers with one and two fine ends, respectively, differed significantly $(P<.001)$ from that in the other compartment.

ments indicate that the finely tapered ends show diametric reduction to $10 \%$ of maximum with 25-50\% (25-30\% for the proximal compartment and $45-50 \%$ for the distal compartment) of the total length so tapered. Perhaps $40 \%$ of each tapered portion still re-
(A), the blunt end (B), and the middle of a fiber (C). $\times 140$.

tains a substantial diameter. Observation during chemical dissection showed that the fibers were not organized end-to-end but rather that their tails overlapped widely.

Four muscles were stained to visualize the motor end-plates along the outside surface of the entire muscle (Fig. 3). One of these muscles was sliced lengthwise, thus visualizing the distribution of motor end-plates in the center of each muscle (Fig. 4). The pattern indicated that the motor end-plates were distributed in bands $(1-3 \mathrm{~mm}$ across the width of the proximal compartment and 2-6 $\mathrm{mm}$ across the width of the distal compartment of the muscle) involving approximately 15-100 motor end-plates placed adjacent to each other. The sliced muscle showed a similar pattern. This suggests that the fibers may be distributed in patches of perhaps 200-1,000 motor end-plates.

The bands of motor end-plates did not seem to cross the muscle from side to side, but rather were staggered. For some sections one seemed to be able to match successive bands at regular intervals along longitudinal strips of muscle; for others one gained the impression that the strips of muscle do not run in 


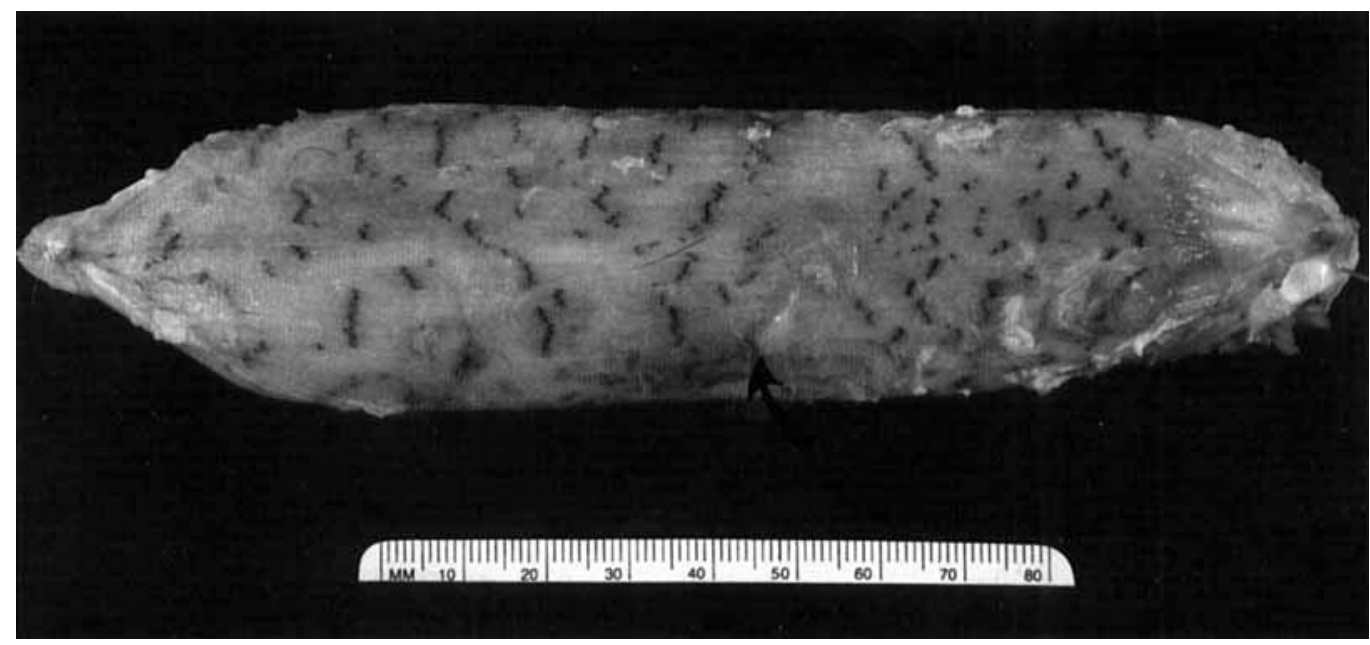

Fig. 3. Capra hircus. Semitendinosus muscle. Acetylcholinesterase stain of muscle surface to show the location and distribution of motor end-plates. The arrow shows the inscription between the proximal (right) and distal (left) bellies. Note that the muscle bands are narrower in the proximal compartment than in the distal compartment.

TABLE 3. Mean values (and ranges) of length $(\mathrm{mm})$ of the tapering section ${ }^{1,2}$

\begin{tabular}{|c|c|c|}
\hline $\begin{array}{l}\text { Fibers and } \\
\text { compartments }\end{array}$ & $\begin{array}{l}\text { Blunt or proximal } \\
\text { end }\end{array}$ & $\begin{array}{l}\text { Fine or distal } \\
\text { end }\end{array}$ \\
\hline \multicolumn{3}{|l|}{ Fibers with 1 blunt end } \\
\hline Proximal & $\begin{array}{c}.343(0.18-.46) \\
N=20\end{array}$ & $\begin{array}{c}6.78(4.97-8.41) \\
\mathrm{N}=20\end{array}$ \\
\hline Distal & $\begin{array}{c}.200(0.142-.284) \\
\mathrm{N}=17\end{array}$ & $\begin{array}{c}8.62(7.31-11.36) \\
\mathrm{N}=17\end{array}$ \\
\hline \multicolumn{3}{|l|}{ Fibers with 2 fine ends } \\
\hline Proximal & $\begin{array}{c}7.27 \quad(5.32-8.73) \\
\mathrm{N}=18\end{array}$ & $\begin{array}{c}7.04(5.95-8.91) \\
\mathrm{N}=18\end{array}$ \\
\hline Distal & $\begin{array}{c}8.15(7.35-8.88) \\
\mathrm{N}=12\end{array}$ & $\begin{array}{c}8.77(7.67-10.08) \\
\mathrm{N}=12\end{array}$ \\
\hline
\end{tabular}

'Data for the same semitendinosus muscle (total length $158 \mathrm{~mm}$ ) of a goat used for Table 2. The proximal compartment measured approximately $70 \%$ that of the distal one. Fibers with one blunt end attached directly to the terminal tendinous inscription ( $\mathrm{N}=12-20)$.

"The mean length of the taper differed significantly between the blunt and tapered sections. For each of these there was a very significant difference $(P<.001)$ between the proximal and the distal compartments. Within each compartment the mean lengths of the finely tapered portions of the fibers did not differ significantly between the distal and the proximal directions. However, there was again a very significant difference between the mean lengths of the tapered portions of fibers in the proximal and distal compartments $(P<.001)$.

parallel. Some of them only lie superficially for part of the length and then pass into the belly of the muscle; others rise out of the muscle mass and sometimes shift laterally.

\section{Electrophysiological mapping}

In the two goats tested electrophysiologically, the proximal compartment was innervated by two quite separate nerve branches of the sciatic. Recordings across the width of the muscle in the first animal confirmed the gross anatomical impression that the more distal deep nerve innervated only the deep part of the muscle and the more proximal superficial nerve was confined to the superficial part, with little overlap (Figs. 5, 6 ). No responses to the stimulation shown in Figure 6 (for arrays $\mathrm{C}$ and $\mathrm{D}$ ) were re- 


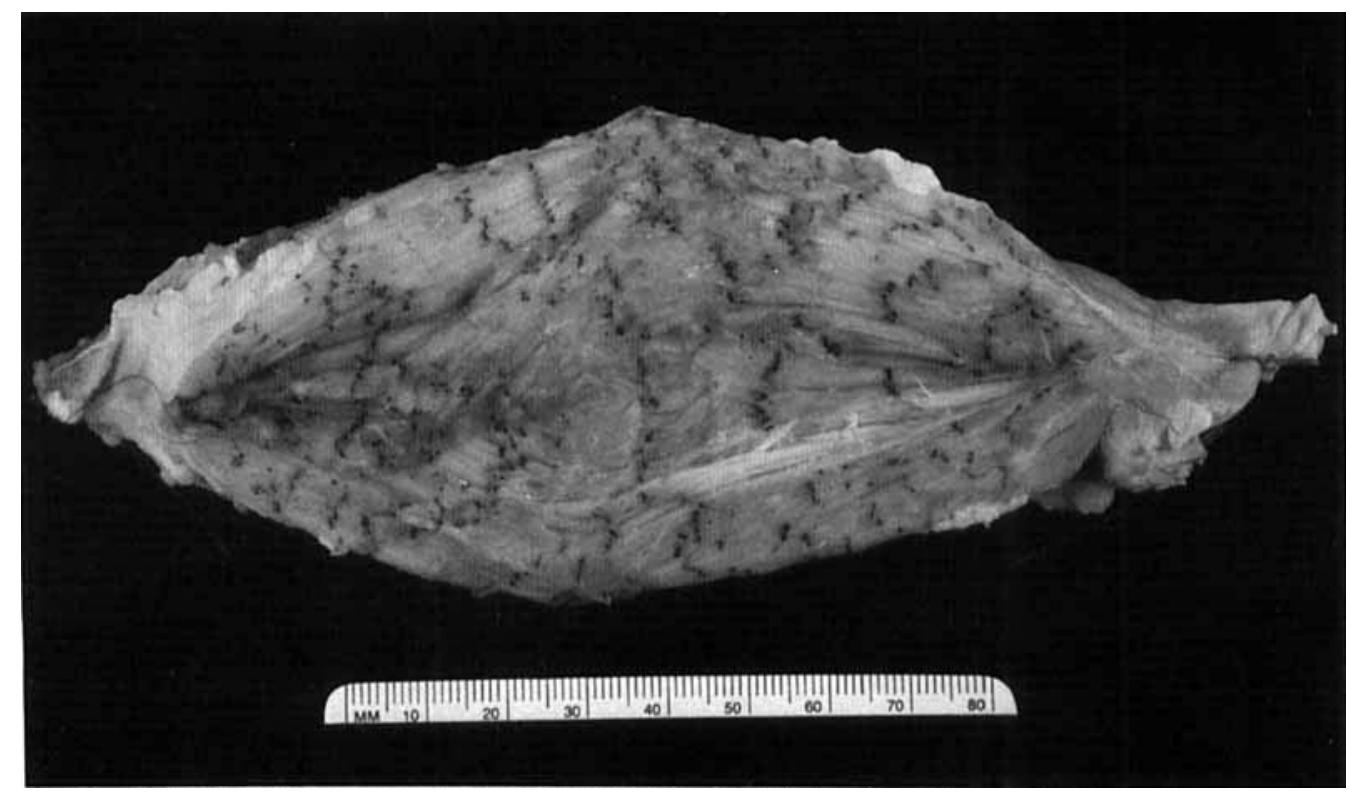

Fig. 4. Capra hircus. Semitendinosus muscle. Acetylcholinesterase stain of muscle that has been split longitudinally to show the location and distribution of motor end-plates within the body of the muscle. The

corded by arrays A and B. The distal compartment was always innervated by one motor nerve which, upon stimulation, evoked potentials across the entire width of the muscle. However, neither nerve appeared to arise from the distal branches of the sciatic nerve; as noted above, they entered the limb parallel to it and then separated.

The polyphasic EMG potentials recorded at each bipolar site appeared to be made of multiple compound action potentials, each having a fairly symmetrical, biphasic waveform. End-plate locations were inferred from reversals in the polarity of the shortest latency component of the waveform in adjacent recording sites. Stimulation of the distal nerve revealed about seven to eight such reversals at irregular intervals over the distal two-thirds of the muscle (see Figs. 5, 6). For 15 such putative end-plate sites in two parallel strips along the muscle, the mean interval between end-plates was 3.4 electrode recording sites with a range of $2-5$. This corresponds to a mean end-plate interval of $10.2 \pm 3.2 \mathrm{~mm}$. Stimulation of the proximal deep nerve produced three putative end-plate sites near the proximal end difference in spacing of fiber bundles in the proximal (right) and distal (left) bellies is obvious. Note that the muscle bands are narrower in the proximal compartment than in the distal compartment.

of the array at similar intervals, although additional end-plates may have been missed in the most proximal $25 \mathrm{~mm}$ of the muscle, which could not be reached by the array. These observations are well correlated with the motor end-plate spacing and the fiber lengths determined by chemical dissection.

Several recordings across four adjacent electrode arrays spaced about 6-7 $\mathrm{mm}$ apart showed some overlap of patterns in adjacent arrays, but the patterns for nonadjacent arrays were quite different (Figs. 5, 6). These seem to match the differential spacing of motor end-plate bands into apparent columns disclosed in the morphological analysis.

As judged from the decrease in amplitude at increasing latency, most of the fibers innervated at any single end-plate site extended only about four to six recording sites in each direction, for a total fiber length of approximately $25-30 \mathrm{~mm}$. However, very small amplitude waveforms at progressively longer latencies could be traced up to 8-11 recording sites away for a few end-plate sites. These usually could be traced in only one direction, but may well have been present in both directions at all end-plates and sim- 
PROXIMAL

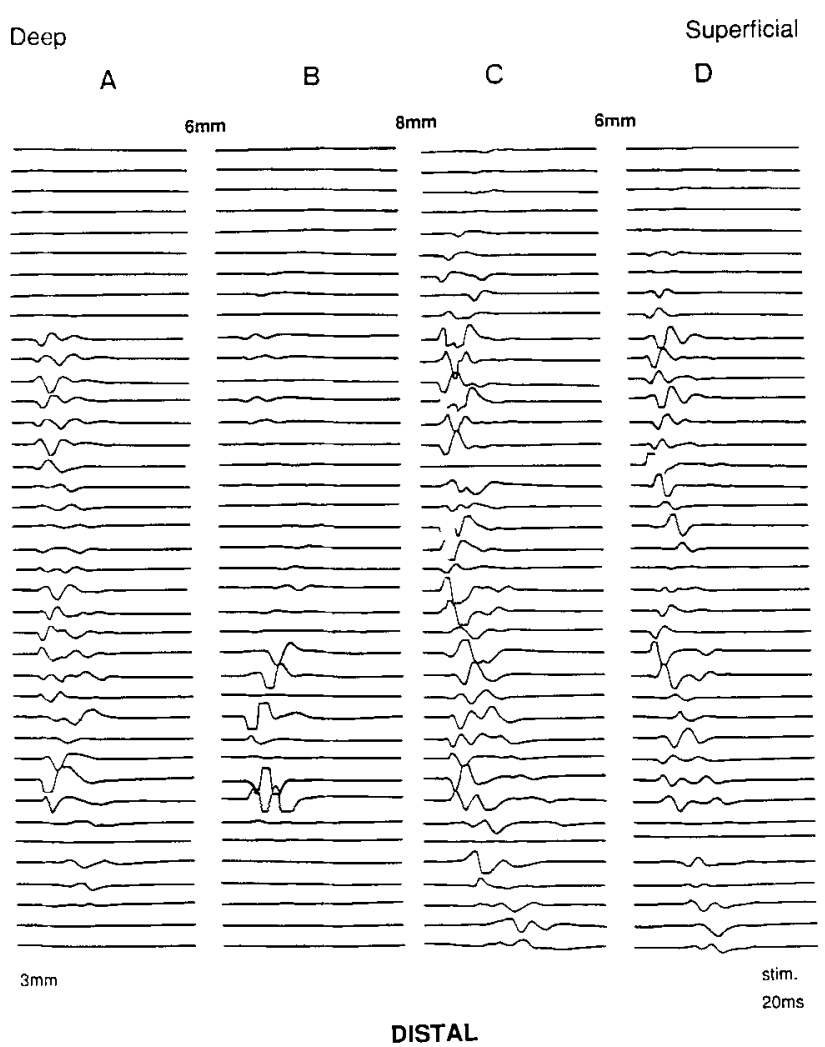

Fig. 5. Capra hircus. Semitendinosus muscle. Composite diagram of EMG traces derived from the recording array and reflecting a composite of 12 equivalent stimulation sessions. The spacing between each vertically separated trace is $3 \mathrm{~mm}$. The numbers along the top $(6,8,6 \mathrm{~mm})$ indicate the physical separation of the recording strips. As the surface of the muscle lies on a plane curving diagonally into the limb, the left side of

ply missed because of the disadvantageous coursing of a small population of long fibers deep into the recording site. When present, the duration and shape of the waveforms were well preserved until they finally disappeared, with no evidence of heterogeneity in conduction velocity (which would cause the waveforms to break up with increasing distance from the innervation point). We infer that these long latency recordings reflect a small population of considerably longer muscle fibers with similar conduction velocity but fiber lengths up to $60 \mathrm{~mm}$ (if they extended symmetrically in both directions from the end-plate region). No such long fibers have yet been detected morphologi- the array lies deeper (and more anteriorly) than the right along the posterior surface of the muscle. See sketch in Figure 7A. The time duration of each trace is $20 \mathrm{msec}$ The entire distal nerve was stimulated at a low level. Note that the most proximal recording sites have little or no activity. Some patterns in adjacent arrays showed some overlap, but those for nonadjacent arrays were quite different.

cally; however, a few fascicles (less than $5 \%$ of the total) appear to pass completely across the proximal-distal inscription barrier (see below). Alternatively, the low amplitude of the long latency signals might reflect decreased action currents from the tapered fibers. Because the muscle nerves were cut proximally and a bipolar cuff confined the stimulus to the distal nerve stump, there is no possible source of such long latency potentials besides conduction delay of action potentials in muscle fibers innervated by that nerve.

By connecting the corresponding peaks in adjacent traces, slope lines (in millimeters distance per millisecond latency) could be es- 


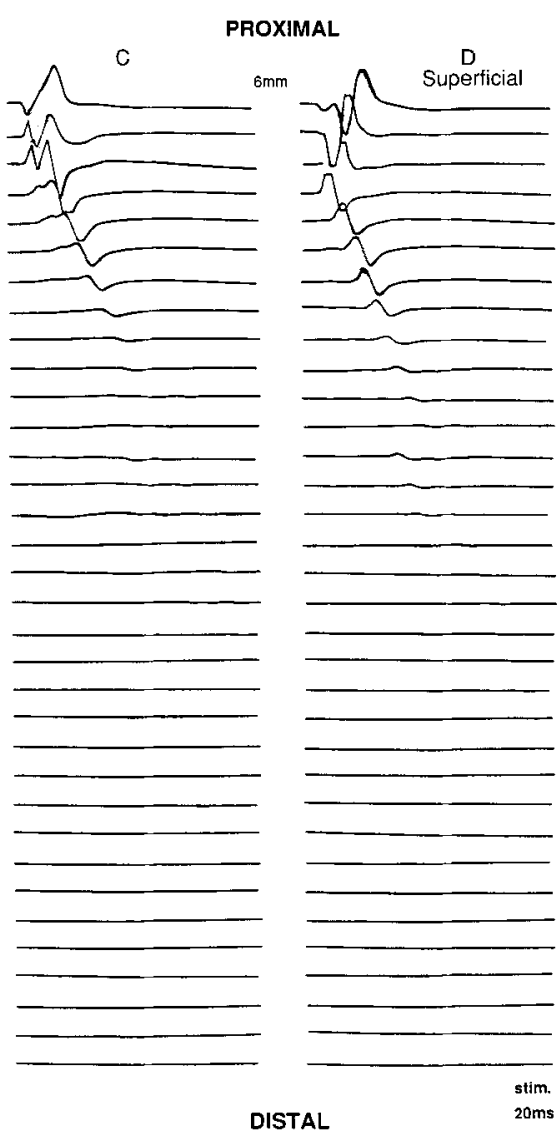

Fig. 6. Capra hircus. Semitendinosus muscle. Composite diagram of EMG traces derived from the same recording array as in Figure 5, but with the entire proximal superficial nerve stimulated. The numbers in the illustration again indicate the physical separation of the recording points. The time duration of each trace is 20 msec. Only the two strips on the right side of the array are shown, as the others lacked signals. Note that the portion of the compartment that shows signals was silent in the record shown in Figure 5.

timated for each subpopulation of fibers innervated at a particular putative end-plate zone (Fig. 7B). These were all clustered in the range of $2.5-3.5 \mathrm{~mm} / \mathrm{msec}(\mathrm{m} / \mathrm{sec})$, with no consistent trends in the proximal vs. distal direction or the site of innervation.

Figure 7C shows the putative sites of endplates and the extent of subpopulations of muscle fibers innervated by the proximal deep and distal nerves in the second animal, as recorded by a single array extending past both nerve entry points, which were $30 \mathrm{~mm}$ apart. Whereas there is no overlap in the putative end-plate sites, there is considerable overlap in the extent of muscle fibers. Part of this may arise from the oblique angulation of the inscription zone that separates these two compartments (not visible grossly for this particular recording situation, but noted by dotted line from these mapping data and from microdissection of muscles from other goats). However, long latency waveforms with small amplitude extended into the most proximal sites upon stimulation of the distal nerve, suggesting that a small slip of muscle fibers may bypass this inscription zone (see asterisk in Fig. 7A), as has been noted in the cat (Loeb et al., '87).

Strain gauge records from the detached distal end of the muscle provided only the crudest approximation of the twitch dynamics, because we activated only one of the two series compartments at a time (Fig. 8). With the muscle passively stretched to about 10 $\mathrm{kg}$ of resting tension, the twitch rise time for stimulation (from onset to maximum force) was approximately $35 \mathrm{msec}$. From the first EMG rise to the start of force generation was $8 \mathrm{msec}$, and it took at least $120 \mathrm{msec}$ for tension to drop back to baseline. True isometric twitches would be expected to be considerably faster. However, near-threshold stimulation of a nerve with mixed motor unit types would be expected to be biased toward the largest diameter, presumably fastesttwitch units. For considerations of mechanical stability, the more relevant number is, perhaps, the $20-80 \%$ rise time, which is 17 msec in this record.

\section{DISCUSSION}

The data from fiber dissection, end-plate staining, and EMG recording all present a consistent picture of the muscle fiber architecture within the long muscle fascicles in each head of the goat semitendinosus muscle. These fascicles are composed of relatively short muscle fibers with finely tapered endings distributed freely within the muscle in an interdigitated, series arrangement. This form of muscle architecture has been recognized only sporadically in the past, but seems likely to be prevalent, if not universal, in muscles with fascicles longer than a few centimeters (reviewed by Loeb et al., '87).

There have been numerous papers describing "muscle fiber length" in which this series-fiber arrangement has been overlooked (e.g., Sacks and Roy, '82); such fiber length descriptions appear to correspond to 


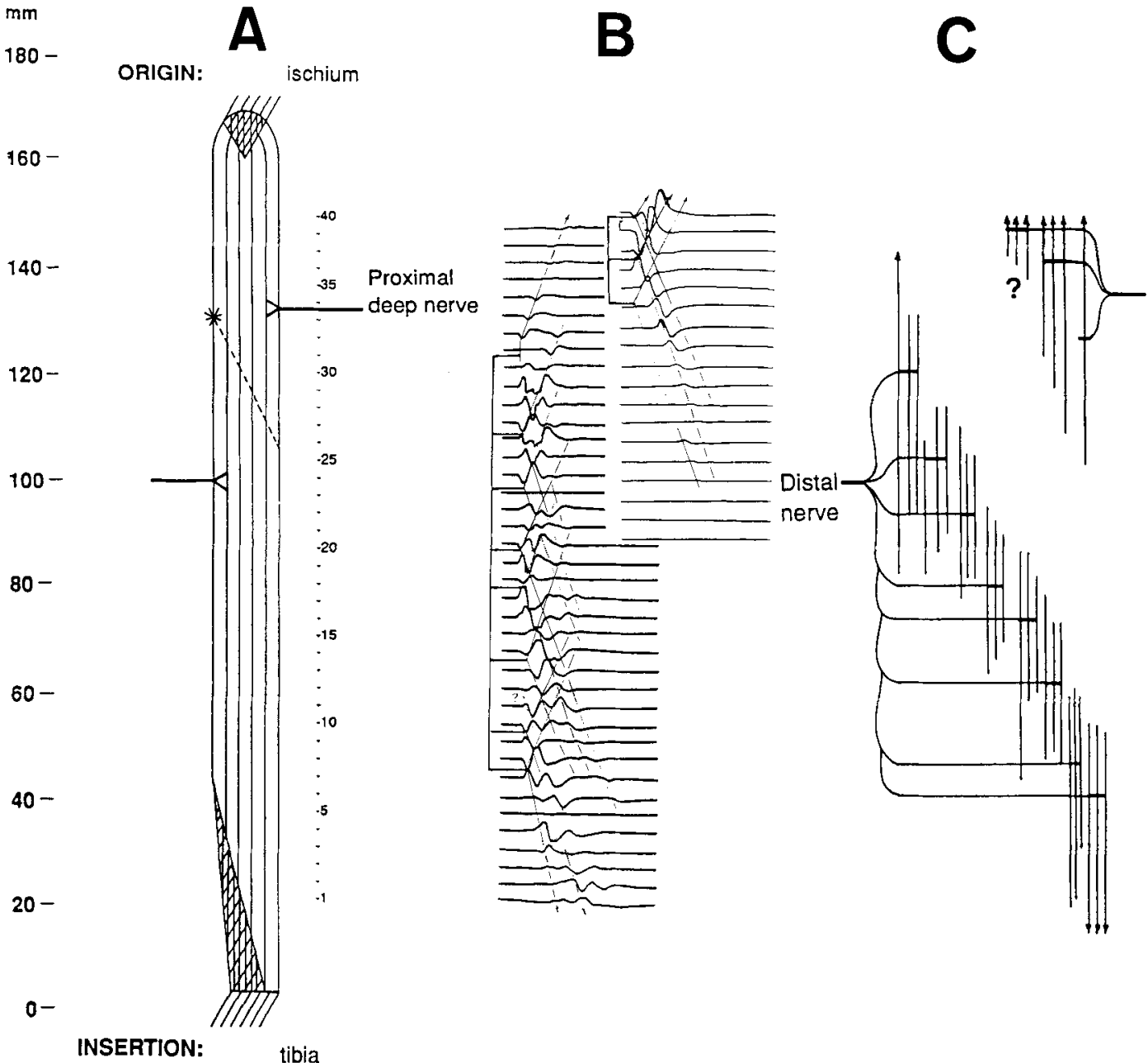

Fig. 7. Capra hircus. Semitendinosus muscle. A: Schematic drawing to scale, showing gross anatomical features of muscle, major nerve entry points, aponeuroses of origin and insertion (cross-hatched), inscription line (dashed line with asterisk), and relative position of sites $1-40$ of the recording array. B: Details from Figures 5 and 6 with the fine, oblique lines indicating corresponding waveform features. End-plates (added horizontal lines connected along the left edge of the raster) are indicated by reversals in polarity of the shortest latency component from negative in one trace to positive in the distal, adjacent trace; these define origins for the

our fascicle length measurements. Indeed, in the dissections described here using similar methods, it was quite possible to miss the long, tapered ends and to conclude that a single, small fascicle was composed of only one long fiber, particularly if connective tissue digestion was less than ideal. For this oblique lines. Dashed portions of the oblique lines indicate probable regions of muscle fiber termination, based upon reduction in EMG amplitude. The waveforms in these regions, although small, have the clearest shapes; where oblique lines cross each other, waveforms are distorted by competing activity. Arrows at the ends of the oblique lines indicate activity extending beyond the electrode array; dotted lines indicate the regions in which the electrical record is unclear, perhaps because of changes in depth of the muscle fascicles. C: Putative distribution of muscle fibers and nerve branches based on the interpretation of records in $B$.

reason, the method of gold staining the muscle fibers (Swash and Fox, '72, as applied to extrafusal muscle by Richmond et al., '85) may be more reliable, because it provides a sharp visual contrast between the darkly stained muscle fibers and the unstained interfiber spaces. 

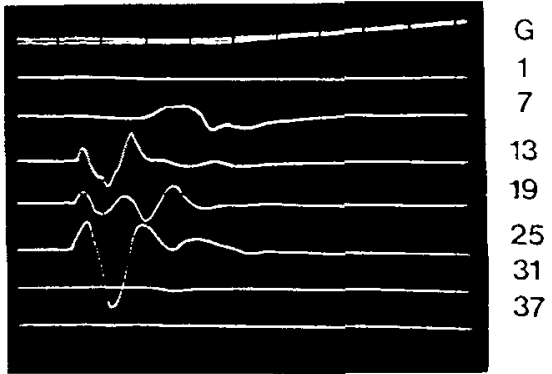

$2 \mathrm{~ms} / \mathrm{div}$
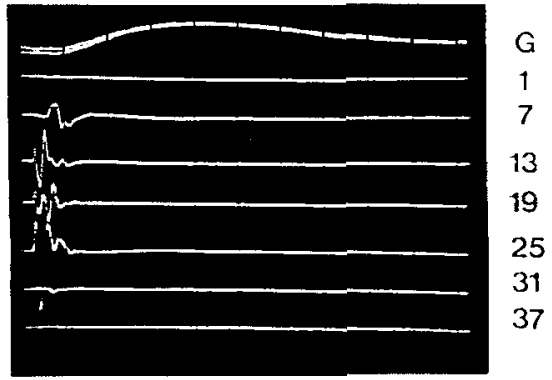

$10 \mathrm{~ms} / \mathrm{div}$.

Fig. 8. Capra hircus. Semitendinosus muscle. Strain gauge output (top trace in each) and the EMGs from four positions. The triggering was set to the time of stimulation; there is a latency of 2 msec until the appearance of the first EMG in any trace. The force begins to rise $8 \mathrm{msec}$ later and peaks (total rise time) at 35 msec. The $20-80 \%$ rise time is $17 \mathrm{msec}$. Top: Time 2 msec per screen grid marking (note top line). Bottom: Time $10 \mathrm{msec}$ per division.

The limited overlap of patterns recorded from adjacent arrays (cf. Figs. 5, 6) suggests that the branching patterns of the nerve are arranged in columnar strips that are no more than $10 \mathrm{~mm}$ wide and extend the length of the compartment. Subdivision into columns would be consistent with the report that single motor axons of the cat bifurcate and innervate muscle fibers distributed in narrow motor territories, resulting in similar crosssectional areas for the unit over the length of the compartment (Loeb et al., '87). The proximal and distal compartments of the semitendinosus muscle appear to be separate muscles innervated by separate motoneurons.

Staining for motor end-plates on the surface and in the center of the muscle provided a pattern equivalent to that in the cat (Loeb

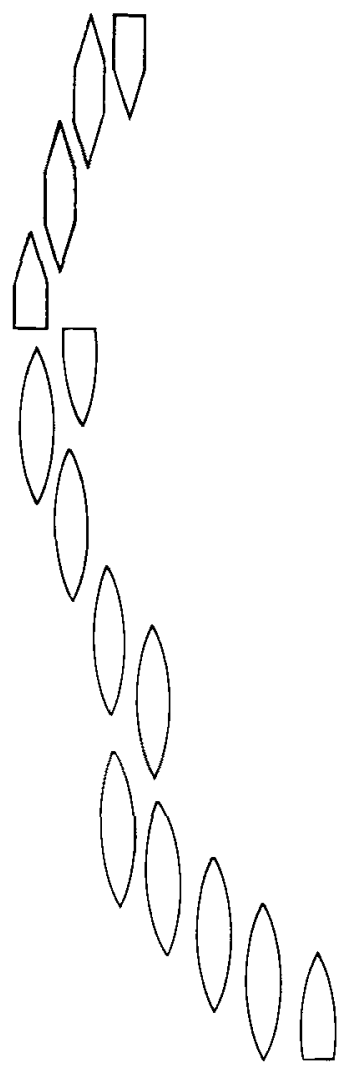

Fig. 9. Capra hircus. Semitendinosus muscle. Reconstruction of the entire muscle to show fiber overlap. The proximal compartment is modeled with four fibers in series, showing approximately $35 \%$ overlap at each end. The distal compartment is modeled with ten fibers in series, showing close to $50 \%$ overlap.

et al., '87). However, the number of motor end-plate bands in series along the length of the muscle was greater, amounting to approximately 14 vs. 5-6 for the cat. This suggests an increase with either mass or length which needs to be tested on a larger sample. As in the cat, the transverse territories did not reach all the way across the muscle, as shown by the transverse staggering of motor end-plate patches. The end-plate staining suggested that the motor end-plate bands were more tightly spaced longitudinally along the proximal rather than the distal compartment; however, this proved to be an error due to greater displacement of the bands, which are narrower in transverse extent in the proximal compartment. The dissolution technique suggests that the fibers are actually slightly longer in the proximal com- 
partment, although our limited sampling cannot rule out regional differences within each compartment.

Calculations attempting to correlate the fiber length with that of the whole muscle suggest that approximately four sets of fibers in substantial overlap comprise the proximal portion of the muscle and ten sets of fibers comprise the distal portion (Fig. 9). The calculations assume that the fibers thus combined had the mean length and that $40 \%$ of each fiber overlapped at one or both ends. These numbers correlate well, both with the staining of end-plate bands and with the physiological data on polarity reversal.

The conduction velocity hypothesis (i.e., the serial short fiber arrangement reflects differential conduction velocities for electrical twitch and mechanical shortening; Loeb et al., '87) does not provide a functional explanation for the terminal thinning and overlap of the muscle fibers. Indeed, as the fibers thin, their membrane conduction velocity might be expected to decrease. Furthermore, the force of the thick belly of the fiber must presumably be transmitted laterally through the sarcolemma and into the surrounding connective tissue matrix rather than be transmitted via the very thin ends. At the moment, the pattern does not suggest a facile, functional explanation. It may well be that the terminal thinning offers opportunity for slippage among the fibers (Rindos et al., '84) or for adjustment of the system during development.

For both the goat and cat, the muscle fiber conduction, the twitch rise time, and the fiber length are similar, with the longer goat muscle being composed of a larger number of similar muscle fibers in series. Thus, the hypothesis that fiber length is limited by conduction velocity is not falsified. The results indicate that the short fiber pattern described for the cat also applies for the goata larger animal and a ruminant rather than a carnivore. The results also suggest that muscle conforms to the rule that cell size does not scale with body size; perhaps this rule is now broken only by neurons (as motoneurons must reach from the central nervous system to the most distal muscle masses in the appendages). One interesting question concerns the innervation ratio of muscle fibers per motoneuron; with many fibers in series for the territory of each muscle unit, either the physiological cross-sectional area of the muscle units must be relatively low or the innervation ratios must be unusually high. Finally, the study clearly documents the congruence of the results obtained by morphological and physiological techniques, which here provided equivalent results leading to further faith in the robustness of the methods.

\section{ACKNOWLEDGMENTS}

It is a pleasure to acknowledge that this is one of a number of studies that could be carried out in part while C.G. held a guestprofessorship at the Department of Biology of the University of Antwerp (UIA). This work was supported by NSF grant G-BSR-850940 (C.G.; with an undergraduate research experience supplement) and FKFO grant 2.9005.84 (F.D.V.). We are grateful to the Zoological Society of Antwerpen and the Detroit Zoological Park for help with the experimental animals. The electrode arrays and amplifiers were provided by Cheryl $M$. Chanaud. Messrs. David Kraklau and Shawn Slywka helped with the staining of motor end-plates and with the determination of fiber length by chemical digestion. David Carrier and Frances J.R. Richmond kindly commented on the manuscript.

\section{LITERATURE CITED}

Chanaud, C.M., C.A. Pratt, and G.E. Loeb (1987) A multiple-contact EMG recording array for mapping single muscle unit territories. J. Neurosci. Methods 21:105112.

English, A.W., and O.I. Weeks (1987) An anatomical and functional analysis of cat biceps femoris and semitendinosus muscles. J. Morphol. 191:161-175.

Gans, C. (1982) Fiber architecture and muscle function. Exercise Sport Sci. Rev. 10:160-207.

Gans, C., and W.J. Bock (1965) The functional significance of muscle architecture: A theoretical analysis. Adv. Anat. Embryol. Cell Biol. 38:115-142.

Gans, C., and F. de Vree (1987) Functional bases of fiber length and angulation in muscle. J. Morphol. 192:6385; erratum, 193:323.

Karnovsky, M.J., and L. Roots (1964) A "direct-coloring" thiocholine method for cholinesterases. J. Histochem. Cytochem. 12:219-221

Loeb, G.E., and C. Gans (1986) Electromyography for Experimentalists. Chicago: The University of Chicago Press.

Loeb, G.E., C.A. Pratt, C.M. Chanaud, and F.J.R. Richmond (1987) Distribution and innervation of short, interdigitated muscle fibers in parallel-fibered muscles of the cat hindlimb. J. Morphol. 191:1-15.

Richmond, F.J.R., D.R.R. MacGillis, and D.A. Scott (1985) Muscle-fiber compartmentalization in cat splenius muscles. J. Neurophysiol. 53:868-885.

Rindos, A.J., G.E. Loeb, F.J. Richmond, and O. Morris (1984) Architectural features of short, series muscle fibers in cat sartorius and tenuissimus muscles. Neurosci. Abstr. 10:629.

Sacks, R.D., and R.R. Roy (1982) Architecture of the hindlimb muscles of cats: Functional significance. J. Morphol. 173:185-195.

Swash, M., and K.P. Fox (1972) Techniques for the demonstration of human muscle spindle innervation in neuromuscular disease. J. Neurol. Sci. 15:291-302. 